\title{
Freight trains and supernovas: the use of a sorting task to determine patterns within long-term frequent attendance to general practitioners
}

Richard D. Neal, Philip L. Heywood Centre for Research in Primary Care, Nuffield Institute for Health, University of Leeds, Leeds and Stephen Morley Division of Psychiatry and Behavioural Sciences in Relation to Medicine, University of Leeds, Leeds, UK

\begin{abstract}
Little is understood about the patterns in which frequent attenders consult primary care over time, or about the clinical or behavioural reasons for doing so. The aim of this study was to use a sorting task to identify the important features of patterns of attendance to general practitioners generated by frequent attenders over a period of 44 months. A total of 12 experts performed a sorting task on consulting patterns of the 100 most frequent attenders from one general practice in Leeds. A similarity matrix was constructed which was then subjected to cluster analysis. A range and diversity of patterns was clearly demonstrated within the data. The determining factors of the groupings by the experts included frequency, regularity, bursts of consultations, patterns of consulting after bursts, periods of not consulting, and seasonal or annual trends. The cluster analysis provided six final clusters, the largest of which had no fixed or classifiable pattern of attendance. The other five clusters were 'regular without bursts', 'supernovas', 'gap and prolonged burst', 'bursts and sporadic attending' and 'regular as clockwork'. Distinct patterns of frequent attendance exist, and it seems likely that the clinical and behavioural reasons underpinning such behaviours differ. This work furthers our understanding of the way in which frequent attenders consult, and the results have implications both for general practice and for future research. The methodology described may have applications in other areas of primary care and biomedical research.
\end{abstract}

Key words: attendance patterns; cluster analysis; frequent attendance; general practice; sorting task

\section{Introduction}

It has been established that a small minority of patients consult primary care services with great frequency and make a significant contribution to the overall clinical workload (Neal et al., 1998). These people are more likely to be female and older (Neal et al., 1998), and receive more prescriptions and referrals than nonfrequent attenders

Address for correspondence: Richard D. Neal, Centre for Research in Primary Care, Nuffield Institute for Health, University of Leeds, 71-75 Clarendon Road, Leeds LS2 9PL, UK.

(C)Arnold 2000
(Svab and Zalatel-Kragelj, 1993; Heywood et al., 1998). However, little is understood about the temporal aspects of frequent attendance. Despite this, most of the published work to date uses a definition based on a fixed number of consultations in a fixed time period (typically 12 consultations over 12 months) (Neal et al., 1996a). Experience from clinical practice and most of the evidence (Semmence, 1969; Ward et al., 1994; Gill et al., 1998; Heywood et al., 1998) suggests that some individuals can drift in and out of frequent consulting over the years, although these studies were limited by narrow 'all or nothing' definitions of frequent attendance. This conflicts with previous

1463-4236(2000)PC006OA 
work which has shown stable consultation rates over a 3-year period (Freer et al., 1986).

The aim of this study was to use a sorting task to identify patterns within the attendances of frequent attenders to general practitioners. By doing this we hoped to determine the extent to which frequent attenders display different patterns over time - for example, showing whether or not patients who consulted frequently over a few months continue to do so for several years, and indeed what other patterns exist. This study is of importance to clinicians in that it will identify long-term patterns of consulting, and through recognition of the pattern of frequent attendance may lead to an understanding of why a minority of patients show the extreme patterns of consulting that they do. It is of importance to the research community in contributing to understanding of the behaviour of frequent attenders in order to develop more meaningful definitions of the frequent attender, and to develop appropriate intervention strategies (Neal et al., 1996a), or other ways of managing demand (Gillam and Pencheon, 1998). Finally, the study uses a novel methodology which is reported in detail here. The use of this methodology and the implications for its use in other primary care research are discussed.

\section{Method}

The study was conducted in one general practice in Leeds, which has an age and sex profile similar to that of the overall UK population (Office of Population Censuses and Surveys, 1991). The collection and validation of these data has been described previously (Neal et al., 1996b), and the practice has a similar profile of consultation rates and frequent attendance to three other local practices (Neal et al., 1998). Visual examination of printouts of general practice consultations over time were plotted ('consultation timelines'). These suggested that discrete and distinct patterns were present. Figure 1 shows the consultation timelines for the 20 most frequent attenders who were continuously registered. Each horizontal row represents an individual's consulting over 44 months, with each data point representing a contact with a GP from that practice. All face-to-face consultations were recorded irrespective of whether they were initiated by doctor or patient, or whether they were for acute conditions or chronic disease management.
Informal input from colleagues suggested that different people interpreted these patterns in different ways. We therefore decided to identify these patterns using a rigorous and valid method. We chose to use an 'expert sorting task', which is a process whereby a group of subjects classifies data (of whatever kind) into groups, and these groupings are then analysed to produce a classification. The expert sorting task retained what we considered to be an important 'human factor' in the analysis, rather than using a solely computer-based clustering model. This methodology has previously been used in a variety of medical settings (Reading et al., 1982; Grober, 1984; Livesley, 1987; Barsky et al., 1993; Crow and Spicer 1995).

A panel of 12 'experts' was recruited, representing professional groups from primary and secondary medical care and higher education. Each expert was given a photocopy of the consultation timelines of the most frequent 100 attenders for the period 1 July 1991 to 28 February 1995 . This period of 44 months was the longest time for which data from this practice were valid (Neal et al., 1996b). The experts were given instructions to cluster the attendance patterns into as many groups as they wished of their own choosing (see Box 1). These instructions were similar to those used in other studies. The 100 most frequent attenders over this time period represented the most frequently consulting $1.5 \%$ of the practice population. Their mean number of consultations was 93.1 (range 69189) (Table 1), and they consisted of 24 males and 76 females, with a mean age of 59.0 years at the end of the study period (range 7-88 years).

The results were collated and a $100 \times 100$ similarity matrix was constructed using a Borland Pascal program (copies of which are available from the authors) to implement Burton's algorithm (Burton, 1972). This matrix was constructed in terms of the number of times each subject was grouped with each of the other 99 subjects. The matrix was then subjected to hierarchical cluster analysis (Everitt, 1993) in exactly the same way as described by Reading et al. (1982). We performed the cluster analysis using various methods of linkage (between groups, centroid, median and Ward's) and measurement between groups (Euclidean and squared Euclidean distance). We then performed a discriminant analysis on the result that provided the best fit.

The cluster analysis works by identifying similar 


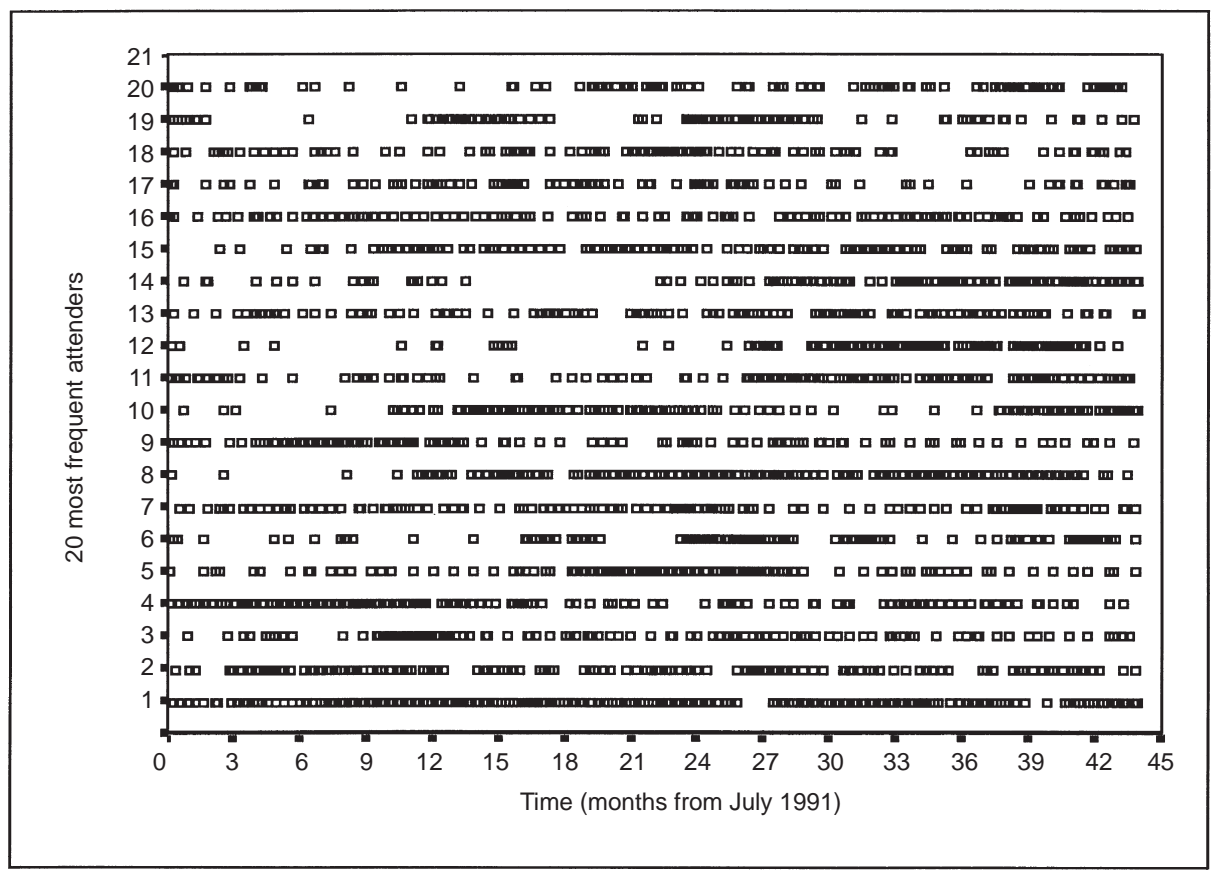

Figure 1 Consultation timelines of the 20 most frequent attenders.

\section{Box 1 Instructions given to each expert}

The aim of this task is to sort 100 frequent attenders at a general practice into groups, all members of which you think have similar characteristics. Examine the 5 sheets and consider whether there are patterns duplicated across patients. Allocate each patient to one and only one pattern according to a rule which you have induced. In addition to allocating patients to groups, please also write out the rule which you have used for that group. Your rule can be as simple or as complex as you like, provided that it is consistent and mutually exclusive (i.e., allocates to that group only).

Please return your judgements to me in the following format:

Group 1: patient numbers, e.g., 21, 43, 65, 78.

Rule for group 1: they were very infrequent attenders.

Many thanks for your co-operation.

subjects (according to a mathematical formula) and grouping the subjects into clusters. Starting from 100 clusters with one subject each, the analysis 'progresses' by producing decreasing numbers of clusters with increasing numbers of subjects, eventually leading to one cluster with 100 subjects. The data were analysed using SPSS for Windows Version 7.0.

The consultation timelines were printed out for each cluster, in order to identify the patterns of 


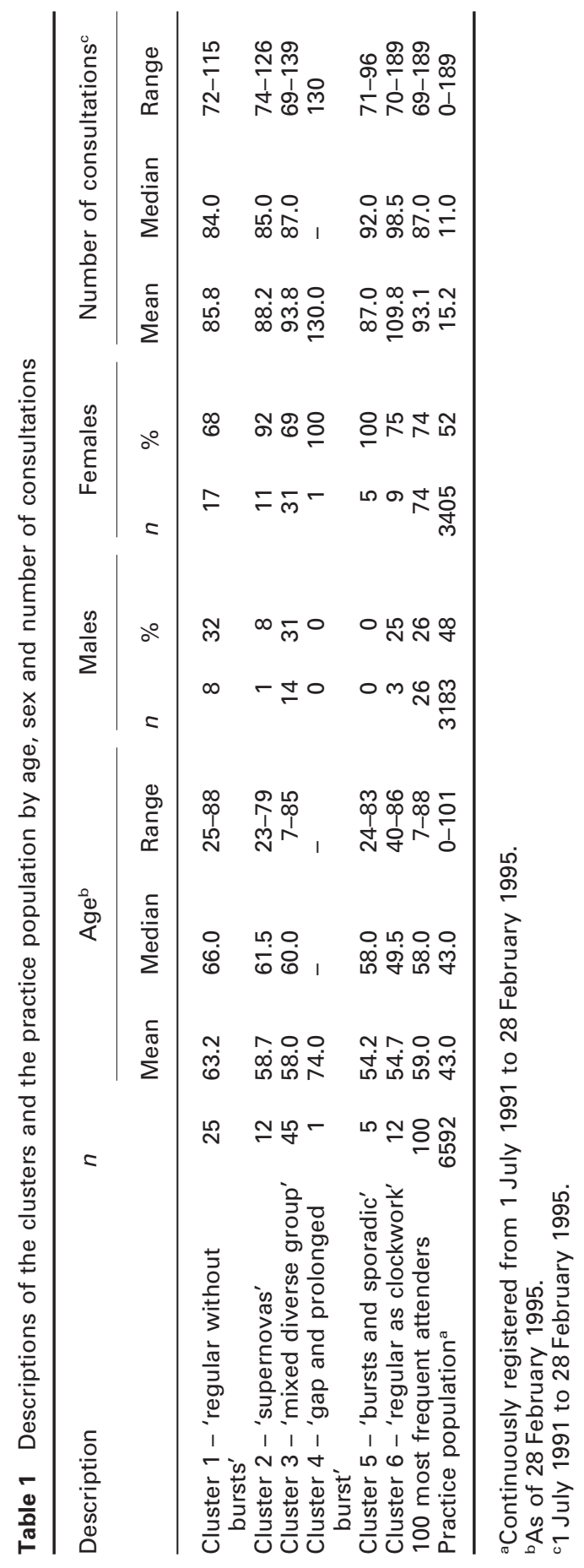

Primary Health Care Research and Development 2000; 1: 39-50 
attendance demonstrated by members of each cluster. Records of the rules for each group supplied by each expert were examined and compared with the final clusters. Consultation timelines for the most frequent attenders in three other practices in Leeds for which we had valid data (Neal et al., 1996b) were then examined to see whether the patterns of attendance were present in these practices.

\section{Results}

\section{The experts' groupings}

The number of groups identified by each expert ranged from 5 to 10 , with a median of 7 . The rules used by the experts, which varied considerably, are shown in Appendix 1. The determining factors included some measure of frequency (e.g., expert 9), some measure of regularity (e.g., experts 2 and 7), bursts of consultations with regard to background consulting, numbers of bursts, and pattern of consulting after bursts (e.g., expert 6), gaps and periods of not consulting (e.g., experts 11 and 3), seasonal or annual trends (e.g., experts 3 and 4), and no fixed or classifiable pattern of attendance (a heterogeneous group) (e.g., experts 2, 7 and 12). The experts varied in their tightness of definition and hence mutual exclusiveness of groups (e.g., experts 2 and 11). Some of them provided descriptive names for the patterns, and others made judgements about the 'causes' of the attendance patterns (e.g., 'in love with doctor', expert 9; 'likes GP' and 'takes holidays', expert 6; 'catastrophe?', expert 8; 'freight train' - a pattern of bursts of consulting interspersed by short and regular gaps akin to the carriages of a freight train, expert 8) or offered interpretation of their patterns (e.g., 'similar usage throughout the whole period with perhaps odd breaks for holidays or periods of hospital admission', expert 4).

\section{The process of clustering}

The 'best fit' from the analysis came from using between-groups linkage with squared Euclidean distance. The 'definitive' result, in terms of the 'correct' number of groups, was decided upon in three ways, as there is no one completely satisfactory technique for doing this (Everitt, 1979):

1) face validity, particularly the determining features of the clusters - see description of the clusters below;
2) examining the way in which the groups were broken down;

3) in such a way that it was in keeping with the numbers of groups suggested by the experts.

The most useful number of clusters to study in this instance was therefore the grouping of six clusters. The discriminant analysis showed that 94 of the 100 subjects fitted into the correct cluster.

The dendrogram in Figure 2 illustrates the process whereby subjects were grouped into clusters. This shows the division into two clusters (subject numbers 1-88 and 89-100), the division into three clusters $(1-83,84-88$ and 89-100), into four (1$82,83,84-88$ and $89-100)$, into five $(1-25,26-$ $82,83,84-88$ and 89-100) and into six (1-25, 26$37,38-82,83,84-88$ and 89-100). Beyond six clusters the number of clusters increases rapidly, with the main difference between the groupings with 6 and 10 clusters being the division of a large group into two, and the creation of discrete clusters containing only one subject. The 'subject numbers' were allocated to each subject after the analysis in order to demonstrate the process of clustering. They do not relate to the frequency of attendance.

\section{The final clusters}

The consultation timelines of the members of the six clusters are shown in Figure 3, and the mean number of consultations by subjects, their mean age and the numbers of males and females in each cluster are shown in Table 1. Figure 3 shows that the clusters were not simply defined by frequency; each cluster contains both frequently and very frequently attending subjects. The age and sex distribution of each cluster were similar. The key determining features of each of the clusters were as follows.

1) Cluster 1 ('regular without bursts') is typified by fairly regular patterns, but also those which tend to fluctuate a little; intense bursts of consulting are absent.

2) Cluster 2 ('supernovas') is typified by prolonged bursts of consultations with fairly low numbers of consultations outside these bursts.

3) Cluster 3 ('mixed diverse group') is typified by the large number of subjects classified into this group, and also by its diversity; there are no similar themes identifiable in the timelines. This is consistent with the fact that most (but 


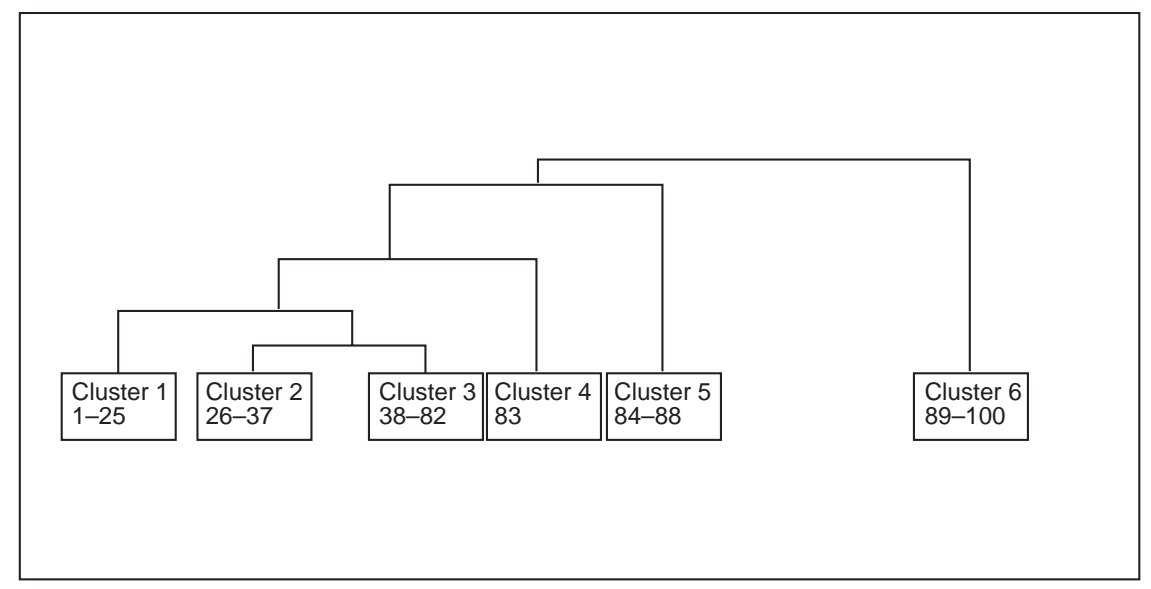

Figure 2 Dendrogram showing the process of clustering (to scale). The subject numbers are numbers allocated to each subject after the analysis in order to demonstrate the process of clustering: they do not relate to the frequency of attendance.

not all) of the experts included a 'mixed' or 'other' group, which was often very large.

4) Cluster 4 ('gap and prolonged burst') contains only one subject who consults very little for the first 18 months of the study, after which he or she consults with alarming regularity, and by doing so is excluded from one of the other categories.

5) Cluster 5 ('bursts and sporadic') is typified by prolonged intense bursts of consultations with minimal numbers of consultations outside these bursts.

6) Cluster 6 ('regular as clockwork') is typified by extremely regular consulting throughout the whole period of study.

Each of the above patterns was found to be present in the consultation timelines of frequent attenders from the other three practices.

\section{Conclusions}

Although the comparison of the rules used by each expert with the final classification suggests that the analysis was meaningful and valid, and that the statistical validity of the clustering was demonstrated by the discriminant analysis, there are potential difficulties with this methodology. A different panel of experts, and data from other practices, may have produced the same or different results. However, the patterns found were present in the other three practices, and our previous research has demonstrated the similarities between practices with regard to their patients with patterns of frequent attendance (Neal et al., 1998). Furthermore, the results do have face validity in that many clinicians will recognize these patterns in their own practices. Indeed, it may be reassuring to clinicians to see that many of the bursts of frequent consulting are short-lived and followed by periods of nonconsulting.

The benefits of using this methodology were that it was a helpful exploratory tool for determining the patterns, it was useful for visually clustering frequent attenders, and it provided an important human context for the study. It proved the value of professional opinion in providing judgements, hypotheses, interpretation, outsider viewpoint and different ways of addressing the patterns of frequent attendance. It would have been possible for a single individual to analyse the consultation timelines and then to make judgements about the presence or absence of certain patterns. The data presented here show that the individuals varied in the way in which they analysed the data, but that the combined analysis of the group provided a way of classifying in a normative way, whilst also being able to encompass something of an individual's analysis into the final classification. The data could have been analysed using only a computer-clustering model. However, this would have involved loss of the researchers' control over the way in 

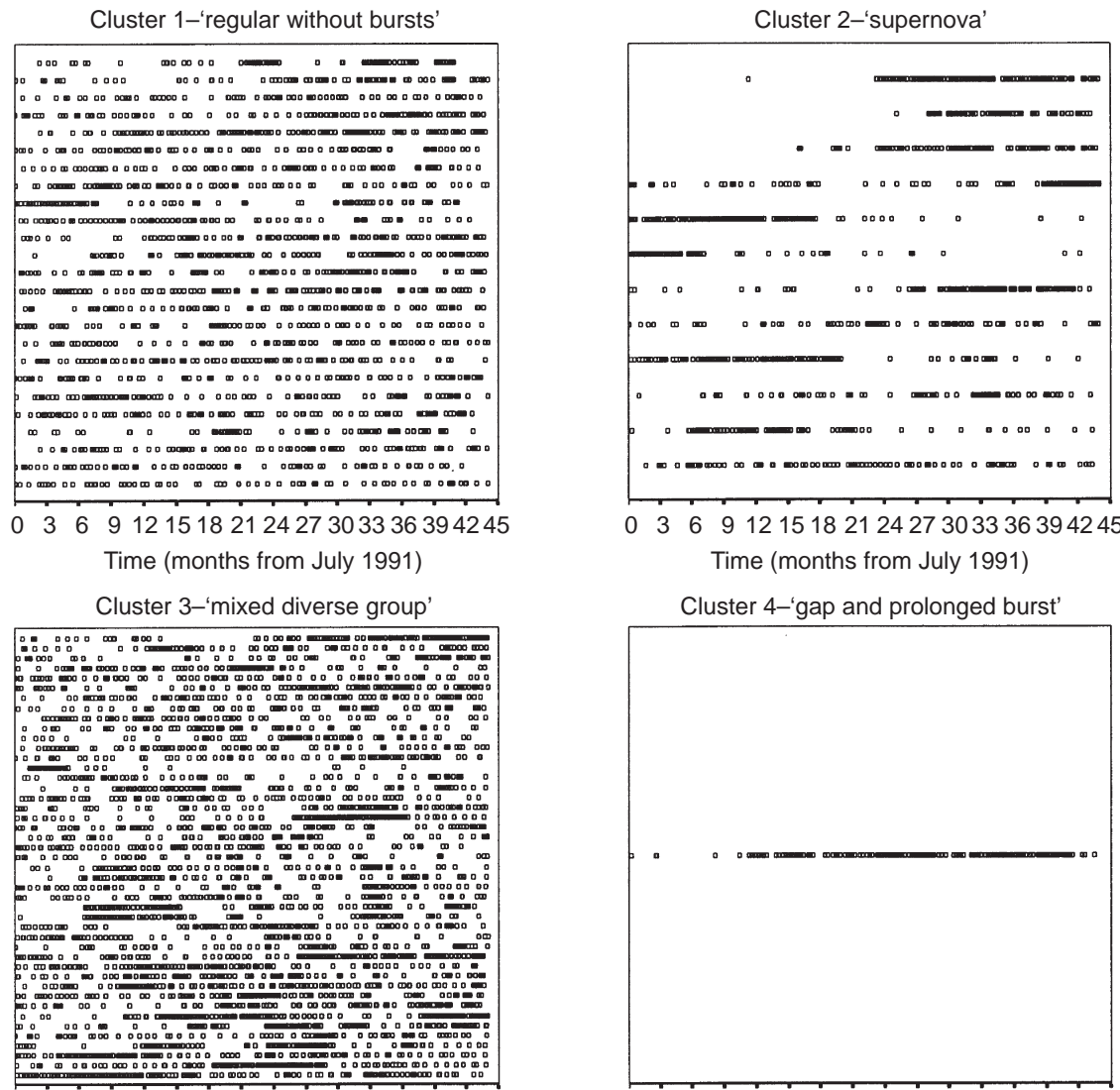

Cluster 4-'gap and prolonged burst'

$0 \quad 3 \quad 6 \quad 9121518212427303336394245$ Time (months from July 1991)

Cluster 5-'bursts and sporadic'
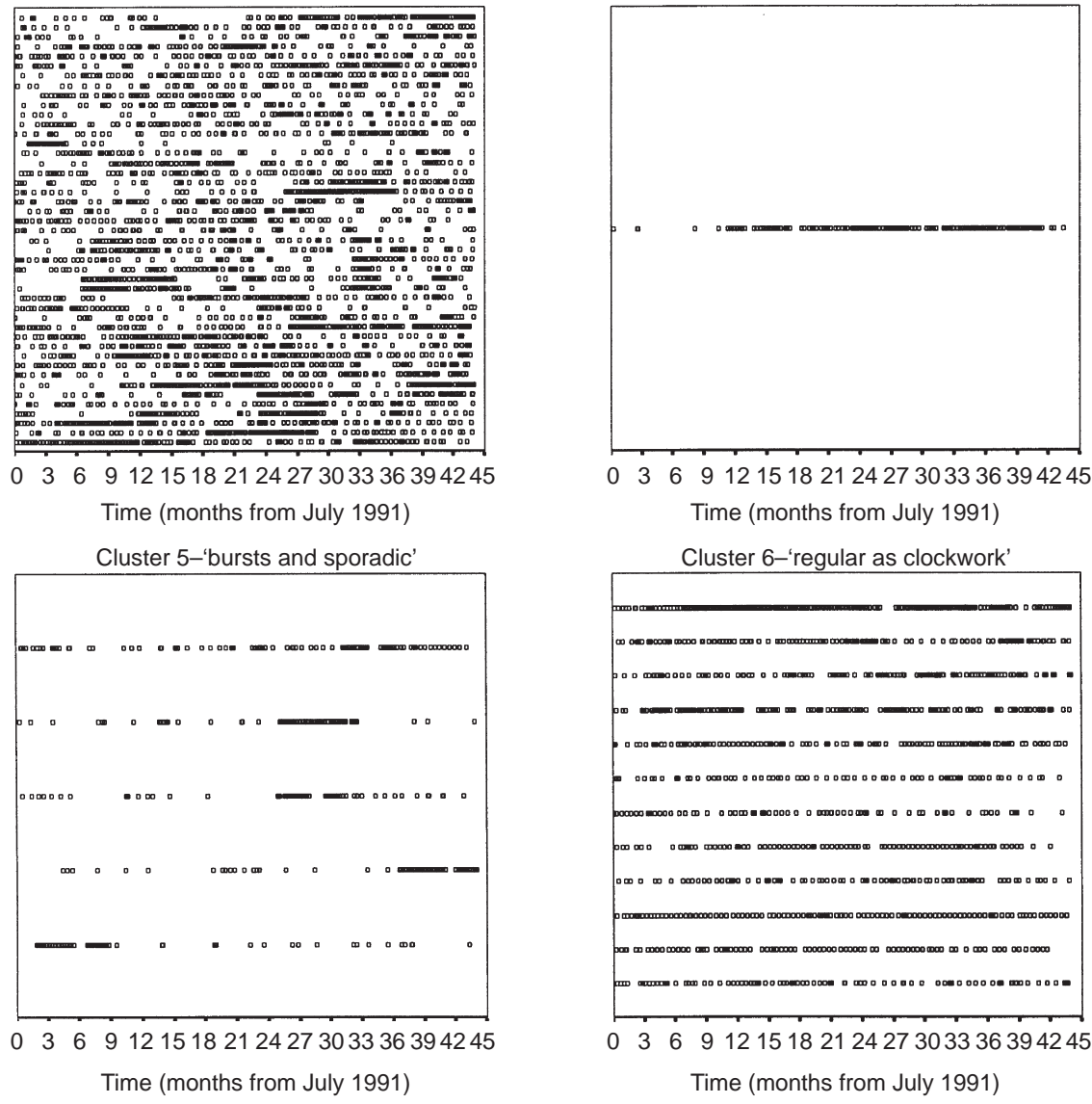

Cluster 6-'regular as clockwork'

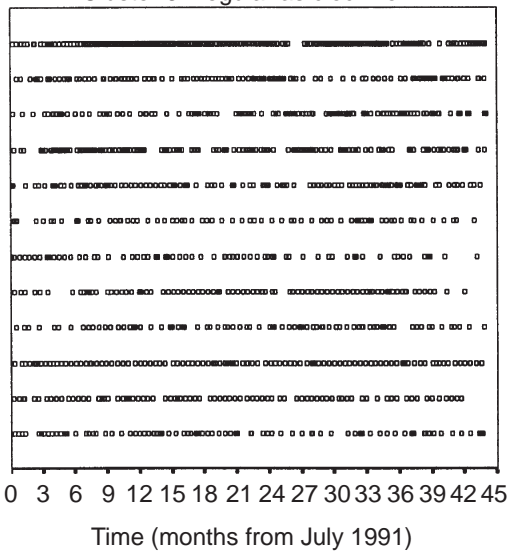

Figure 3 Consultation timelines of the members of the six clusters. 
which the data were classified or which types of patterns were being sought. We are unaware of any instance of such a methodology being used before in primary care research, or to classify patterns of attendance. We believe that this methodology may have applications in other primary care or biomedical research, including recognition of patterns of disease or symptom occurrence, and attendance patterns in other settings.

The main finding of this study is that, although one of the main determinants of the pattern of frequent attendance is the frequency of consultation, other important determinants include the regularity of attendance, or specific irregularities which are typified by bursts of consultations, and gaps during which there are few or no attendances. However, a large proportion of the patterns were grouped together as a diverse 'mixed' category, suggesting that those who display more distinctive patterns are in the minority, and that the patterns represent a spectrum of behaviour ranging from patients who consult with extreme regularity to those who consult in intense bursts with minimal consulting in between those bursts.

This research furthers our understanding of frequent attendance by demonstrating the temporal patterns of consulting over a period of several years. It shows that the natural history of frequent attendance is unpredictable, and that any 'snapshot' of, for example, individuals who make 12 consultations over 12 months, will include people who have consulted very frequently prior to this period together with those who have attended very little if at all. Similarly, it will include patients who are coming to the end of their episode of consulting, together with those who will continue to consult.

An understanding of the multi-factorial and complex reasons why people consult (Campbell and Roland, 1996) means that the finding of the large diverse group comes as no surprise. Possible clinical and behavioural reasons for both the 'supernova' and the 'regular as clockwork' patterns may require more detailed explanation. Although many individuals experience acute illnesses that require frequent consultations over a period of time, we were surprised by the extremity of some of these patterns, and we wonder what the justification for them can be. Similarly, many individuals suffer from chronic illnesses which need regular medical input. However, we were again surprised by the extreme and prolonged regularity of some of this consulting, and we question what the role of the general practitioner may be in prolonging, initiating and perpetuating such patterns of behaviour. Further investigation of these patterns and exploration of the reasons behind them, from the perspective of both doctors and patients, is needed if the phenomenon of frequent attendance is to be understood and appropriately addressed. The clinical relevance of this study can only be judged once this work has been done.

\section{Acknowledgements}

We would like to thank all the experts for their co-operation with this study, the practice for allowing us access to their data, Brett Scaife for a statistical review of the paper, Robert Hills of the University of Leeds Computing Service for help in running the program to generate the similarity matrix, and Kevin Appleton for comments on an earlier draft of the paper.

The study was funded by the NHS Executive Northern and Yorkshire Regional Office through a Research Training Fellowship to R.D.N. The views expressed here are those of the authors and not necessarily those of the NHS Executive.

\section{References}

Barsky, A.J., Coeytaux, R.R., Sarnie, M.K. and Cleary, P.D. 1993: Hypochondriacal patients' beliefs about good health. American Journal of Psychiatry 150, 1085-89.

Burton, M.L. 1972: Semantic dimensions of occupational names. In Romney, A.K., Shepard, R.N. and Nerlove, B., editors. 1972: Multidimensional scaling. Volume II. New York: Seminar Press, 55-72.

Campbell, S.M. and Roland, M.O. 1996: Why do people consult the doctor? Family Practice 13, 75-83.

Crow, R. and Spicer, J. 1995: Categorisation of the patient's medical condition - an analysis of nursing judgement. International Journal of Nursing Studies 32, 413-22.

Everitt, B.S. 1979: Unresolved problems in cluster analysis. Biometrics 35, 169-81.

Everitt, B.S. 1993: Cluster analysis, 3rd edn. London: Edward Arnold.

Freer, C.B., Boyle, P. and Ryan, M.P. 1986: A study of attendance patterns in general practice over three years. Health Bulletin (Edinburgh) 44, 75-81.

Gill, D., Dawes, M., Sharpe, M. and Mayou, R. 1998: GP 
frequent consulters: their prevalence, natural history, and contribution to rising workload. British Journal of General Practice 48, 1856-57.

Gillam, S. and Pencheon, D. 1998: Managing demand in general practice. British Medical Journal 316, 895-98.

Grober, E. 1984: The breakdown of word meanings in aphasia. Cortex 20, 557-66.

Heywood, P.L., Cameron Blackie, G., Cameron, I.H. and Dowell, A.C. 1998: An assessment of the attributes of frequent attenders to general practice. Family Practice 15, 198-204.

Livesley, W.J. 1987: A systematic approach to the delineation of personality disorders. American Journal of Psychiatry 144, $772-77$.

Neal, R.D., Dowell, A.C., Heywood, P.L. and Morley, S. 1996a: Frequent attenders - who needs treatment? British Journal of General Practice 46, 131-32.

Neal, R.D., Heywood, P.L. and Morley, S. 1996b: Real world data - retrieval and validation of consultation data from four general practices. Family Practice 13, 455-61.
Neal, R.D., Heywood, P.L., Morley, S., Clayden, A.D. and Dowell, A.C. 1998: Frequency of patients' consulting in general practice and workload generated by frequent attenders; comparisons between practices. British Journal of General Practice 48, 895-98.

Office of Population Censuses and Surveys 1991: General household survey. London: HMSO.

Reading, A.E., Everitt, B.S. and Sledmere, C.M. 1982: The McGill Pain Questionnaire: a replication of its construction. British Journal of Clinical Psychology 21, 339-49.

Semmence, A. 1969: Chronic high users in a general practice. Journal of the Royal College of General Practitioners 17, 304-10.

Svab, I. and Zalatel-Kragelj, L. 1993: Frequent attenders in general practice: a study from Slovenia. Scandinavian Journal of Primary Health Care 11, 38-43.

Ward, A.M., Underwood, P., Fatovich, B. and Wood, A. 1994: Stability of attendance in general practice. Family Practice 11, 431-37. 


\section{Appendix 1 Definitions of groups by experts}

Definitions of groups

Number in

each group

Expert No. 1

1) Very very frequent attender - regular type $\quad 8$

2) Very very frequent attender - irregular type 12

3) Very frequent attender - regular type 12

4) Rapid burst attender - VFA supernova ['VFA' = very frequent attender] 4

5) Irregular VFA $\quad 64$

\section{Expert No. 2}

1) Regular as clockwork or almost regular throughout time 14

2) Very regular ... less regular ... very regular (or combination) 10

3) Loads ... none ... loads (or combination)

4) Sudden heavy burst(s) $\quad 8$

5) Regular but with gaps $\quad 3$

6) Mixed 29

7) Fluctuatingly regular 29

\section{Expert No. 3}

1) No quarters without consultation, and no variation between quarters in the 27 number of consultations (by eye)

2) No quarters without consultation, and only one quarter with intensive consultation 18 (by eye)

3) No quarters without consultation, and two consecutive quarters with intensive 4 consultation

4) As group 3, but three or more quarters with intensive consultation 10

5) One blank quarter (no consultations) and 'significant' variation between the other 25 quarters (by eye)

6) One blank quarter, and no significant variation between the other quarters 6

7) At least one period of two consecutive blank quarters 5

8) At least three consecutive blank quarters $\quad 5$

\section{Expert No. 4}

1) The 'bomb' - a single period/episode/explosion of high use against a background 24 of lesser/normal use

2) The 'starting pistol' - initial period of lesser/normal use, followed by period of 7 higher use, followed by a period of continuous higher than average use

3) The 'annual burst' - seasonal high or low use (e.g. every winter/spring); 6 increased frequency of attendance will be noted at the same time each year

4) The 'Roman candle' - an intermittent non-seasonal burster - explosive periods of high use against a background of lesser/normal use

5) The 'waterfall' - a constant attender - similar usage throughout the whole period 26 (perhaps with odd breaks for holidays or periods of hospital admission)

6) 'Chaos' - an apparent lack of pattern

\section{Expert No. 5}

1) Steady frequent attender $\quad 37$

2) An initial burst of frequent attendance $\quad 13$

Primary Health Care Research and Development 2000; 1: 39-50 
3) A middle burst of frequent attendance $r$

4) A late burst of frequent attendance 12

5) Bursts of frequent attendance (no more than two) 23

6) Bursts of frequent attendance, but now low attendance rate 3

7) Frequent attendance, new but previously low attendance 4

\section{Expert No. 6}

1) Starter 14

2) Burster 10

3) Letting go 1

4) Fluctuater 12

5) Hop start 3

6) Takes holidays 21

7) Likes GP 39

\section{Expert No. 7}

1) Regular and frequent 9

2) Very frequent 2

3) Intermittently frequent $\quad 7$

4) Irregular and frequent 1

$\begin{array}{ll}\text { 5) Others } & 75\end{array}$

\section{Expert No. 8}

1) 'Continuous' - no breaks 1

2) 'Necklace' - evenly spread, regular rhythm 15

3) 'Freight trains' - frequent, but in bursts with few consultations in between 17

4) 'Starbursts' - very frequent indeed, separated by very long intervals of low 14 frequency

5) 'Big bang' - normally infrequent, one burst of very frequent consultations 10

6) 'Hazard white lines' - very frequent but occasional breaks in regular pattern 15

7) 'Always frequent' - one or two very frequent bursts 21

8) 'Transition' - normally low frequency, moving into or out of very frequent 7 pattern (?catastrophe)

\section{Expert No. 9}

1) Fairly frequent, steady 33

2) Fairly frequent, with patches of very frequent 18

3) Fairly frequent, with patches of infrequent 15

4) Fairly frequent, with patches of very frequent and patches of infrequent 22

5) Half and half-pattern changes from one type of behaviour to another (either 11 infrequent/fairly frequent, or infrequent/very frequent)

6) In love with doctor!

\section{Expert No. 10}

1) Regularly on a continuous basis throughout the time, high frequency (about every 9 month or more), absence of discrete episodes of increased attendance

2) Regularly on a continuous basis throughout the time, high frequency (about every 6 month or more), presence of discrete episodes of increased attendance

3) Regularly on a continuous basis throughout the time, low frequency (about every 31 3 months or so), absence of discrete episodes of increased attendance 
4) Regularly on a continuous basis throughout the time, low frequency (about every 19 3 months or so), presence of discrete episodes of increased attendance

5) Irregular, one episode of high attendance

6) Irregular, two or more discrete episodes of high attendance 12

7) Irregular, irregularly

\section{Expert No. 11}

1) 0 gaps of $\geqslant 45$ days $\quad 7$

2) 1 gaps of $\geqslant 45$ days 11

3) 2 gaps of $\geqslant 45$ days 15

4) 3 gaps of $\geqslant 45$ days 10

5) 4 gaps of $\geqslant 45$ days 14

6) 5 gaps of $\geqslant 45$ days 11

7) 6 gaps of $\geqslant 45$ days 10

8) 7 gaps of $\geqslant 45$ days $\quad 8$

9) 8 gaps of $\geqslant 45$ days $\quad 8$

10) $>8$ gaps of 45 days $\quad 6$

(45 days chosen as half of one division on the plot, and is longer than most patients' and doctors' holidays)

\section{Expert No. 12}

$\begin{array}{ll}\text { 1) Very frequent } & 7\end{array}$

2) Irregular $\quad 3$

3) Regular $\quad 12$

4) Sporadic $\quad 3$

$\begin{array}{lr}\text { 5) Others } & 75\end{array}$ 